\title{
Modeling Category-level Purchase Timing with Brand-level Marketing Variables
}

\author{
Dennis Fok* \\ Econometric Institute \& ERIM \\ Erasmus University Rotterdam
}

\author{
Richard Paap \\ Econometric Institute \\ Erasmus University Rotterdam
}

Econometric Institute Report EI 2003-15

\begin{abstract}
Purchase timing of households is usually modeled at the category level. Marketing efforts are however only available at the brand level. Hence, to describe category-level interpurchase times using marketing efforts one has to construct a category-level measure of marketing efforts from the marketing mix of individual brands. In this paper we discuss two standard approaches suggested in the literature to solve this problem, that is, using individual choice shares as weights to average the marketing mix, and the inclusive value approach. Additionally, we propose three alternative novel solutions, which have less limitations than the two standard approaches. The new approaches use brand preferences following from a brand choice model to capture the relevance of the marketing mix of individual brands. One of these approaches integrates the purchase timing model with a brand preference model.

To empirically compare the two standard and the three new approaches, we consider household scanner data in three product categories. One of the main conclusions is that the inclusive value approach performs worse than the other approaches. This holds in-sample as well as out-of-sample. The performance of the individual choice share approach is best unless one allows for unobserved heterogeneity in the brand choice models, in which case the three new approaches based on modeled brand preferences are superior.
\end{abstract}

*We thank Philip Hans Franses and participants of the Marketing Science conference 2002 in Alberta for helpful comments. All calculations are done using Ox 3.20 (Doornik, 1999). Address for correspondence: D. Fok, Econometric Institute H11-2, Erasmus University Rotterdam, P.O.Box 1738, NL-3000 DR Rotterdam, The Netherlands, e-mail: dfok@few.eur.nl 


\section{Introduction}

To describe purchase timing several models are proposed in the literature, see Seetharaman and Chintagunta (2003) for a recent overview. One usually aims at describing the relation between interpurchase times and various explanatory variables. These explanatory variables can be divided into two groups. The first group corresponds to householdspecific variables, like household size and family income, but also variables as the current stock of the product and the time since last purchase within the product category. These variables can be directly linked to the interpurchase times. The second group contains marketing-mix variables, like price and the presence of promotional activities. These variables cannot be directly linked to the interpurchase times, as marketing-mix variables are observed at the brand level and purchase timing is modeled at the category level.

In the ideal case, we would have knowledge of the preferred brand of each household at every moment in time. To explain purchase timing we could then use the marketing mix of the brand that is bought or would be bought at any moment in time. In practice this is of course infeasible. First of all the data collection would be practically impossible. Second, the household may not have a unique preferred brand at every point in time. It is therefore up to the researcher to somehow summarize the marketing efforts of all brands into category-level indices. This task is exactly the research question we address in this paper. The key question can be summarized as

\section{- What to do with the marketing mix when modeling purchase timing? -}

or in other words: how to construct a category-level measure of marketing efforts from the marketing mix of individual brands that can be included in a category-level interpurchase time model?

One may think that the answer to this question is to use the marketing mix of the purchased brand. There are however two major problems with this approach. First of all, in the decision process of a household, the purchase timing decision precedes the brand choice decision. To the researcher, knowledge of the purchased brand therefore includes the information that a purchase is made in the category. Technically speaking, one therefore cannot use information on the purchased brand to construct explanatory variables for a purchase timing model. A second problem is that brand choice is not available at nonpurchase moments. One may opt to use the marketing mix of the previously purchased brand, but this is likely to be sub-optimal as households may switch brands. In fact, a household may change preferences several times in between two purchases, especially if 
the marketing mix changes in this period.

We are of course not the first to notice these problems in modeling interpurchase time. In every purchase timing study the researcher will have to decide upon how to construct category-level marketing-mix variables. An often-used solution is to use a weighted average of brand specific marketing-mix variables. The weights are usually household specific and obtained from choice shares of the particular household, see for example Gupta (1988, 1991). A disadvantage of weighting the marketing mix using choice shares is that household-specific information is required to obtain the weights. This approach is therefore less suitable for out-of-sample forecasting. Finally, as choice shares are by definition constant over (periods) of time the model does not take into account that preferences may change over time.

Another popular approach amounts to using the so-called inclusive value from a brand choice model as a summary statistic for the marketing efforts in a category, see among others Bucklin and Gupta (1992), Chintagunta and Prasad (1998) and Bell et al. (1999). The inclusive value has the interpretation of the expected maximum utility over all brands in the category. The inclusive value naturally depends on the marketing mix of all brands. A large expected utility is expected to be positively correlated with the probability of a purchase in the category. Although theoretically appealing, this specification is rather restrictive. In the corresponding purchase timing model there is only one parameter that relates all marketing efforts of all brands to the purchase timing, that is, the coefficient corresponding to the inclusive value. Moreover the effects of marketing variables are restricted to be more or less the same on choice as on purchase timing. Another problem may be that the relation between the inclusive value and purchase incidence may only hold within households. Between households there may be substantial differences in inclusive value that are not related to differences in purchase timing. A household with a strong brand preference may have a larger inclusive value than a household with less pronounced preferences. Of course, one cannot conclude from this that the first household will on average have shorter interpurchase times. The between-household differences will be even more pronounced when the brand choice model allows for unobserved heterogeneity in brand preferences.

To meet the limitations of the above-mentioned solutions, we introduce in this paper some alternative specifications. The idea behind these specifications is to use brand choice probabilities as indicators of brand preferences. One method to summarize the marketing efforts of all brands to the category level is again to use a weighted average of the marketing 
mix of each brand, but now using the current preferences of the household as weights. This approach is very similar to using choice shares as weights as in Gupta $(1988,1991)$. However, in our case the preference weights may change over time as they are captured by a brand choice model. Another method is to specify brand-specific purchase incidence probabilities, or, in a continuous model, brand-specific hazard functions. The category purchase probability is then obtained as the weighted average of these probabilities using preference probabilities.

Although these solutions meet the limitations of the standard approaches in the literature, they still consider brand choice and purchase timing as separate issues. However, the fact that a household does not make a purchase in a particular week, reveals information about the preferences of this household. For example, consider the situation where a household frequently purchases a certain brand that is also frequently promoted. Assume that this household never purchases other brands when they are promoted. If one only considers purchase occasions one may overestimate the effect of promotions on brand choice as the non-purchase promotional activities are completely ignored. The fact that the household does not purchase the other brands while they are promoted implies that it has a strong base preference for the frequently purchased brand. It would therefore be better to integrate the interpurchase time model with a brand choice model. In this model the brand choice of households are revealed at purchase occasions, while at non-purchase occasions the preferred brand is treated as a latent (unobserved) variable. In this way, we also use information revealed by households at non-purchase occasions to model brand choices and interpurchase timing. We will call this specification the latent preferences purchase timing model.

To answer the question concerning the inclusion of the marketing mix, we consider the two standard approaches (based on choice shares and based on the inclusive value) and compare them with our two alternative approaches (based on brand choice probabilities) and the latent preference model. In Section 2 we discuss the statistical differences of the various model specifications and we discuss parameter estimation. The analysis is done using a continuous time hazard specification but can easily be adjusted to the discrete case, where one models purchase incidence using binary logit models. In Section 3 we compare different specifications using data on purchases in three product categories. The comparison is based on in-sample fit and out-of-sample forecasting performance. Finally, in Section 4 we conclude. In this section we also discuss the practical implications for modeling purchase timing. 


\section{Modeling interpurchase timing}

One of the most popular models to describe duration data is the hazard model. This model is also frequently used to describe purchase timing at the category level, see for example Jain and Vilcassim (1991), Vilcassim and Jain (1991), Helsen and Schmittlein (1993) and Chintagunta and Haldar (1998) among many others. The marketing-mix variables which are used to explain purchase timing are measured at the brand level. In this section we propose several solutions to incorporate explanatory variables that are measured at the brand level in a category-level hazard model. To highlight the differences between the solutions we first have to introduce some notation. Although the notation may sometimes be complex, it is not necessary to fully understand the mathematics to follow the ideas in this paper.

Denote by $d_{\text {in }}$ the purchase timing of the $n$-th purchase of household $i$ in calendar time, $n=0, \ldots, N_{i}$. The $N_{i}$ observed interpurchase times are therefore defined as $t_{i n}=$ $d_{i n}-d_{i, n-1}$, with $n=1, \ldots, N_{i}$. Note that $t$ refers to the time in a particular duration. The value of $t$ is set to 0 at the beginning of each duration. We assume that the marketingmix variables are constant within one week, which leads to the natural assumption that the brand choice preferences of households are constant within a week. Denote by $\tau_{l}$, $l=1, \ldots, L$, the time indices of a change in the covariates. Using this notation, week 1 corresponds to the interval $\left[\tau_{0}, \tau_{1}\right]$. Furthermore, denote by $K_{i n}(t)$ the week number corresponding to $t$ time periods after the start of the $n$-th interpurchase spell. Note that after a purchase a new "week" will start. In Figure 1 we give a graphical representation of the purchase process. In this example we have purchases in weeks 2 and 4 , and in this case we would have $K_{\text {in }}(0)=2$ and $K_{\text {in }}\left(t_{i n}\right)=4$.

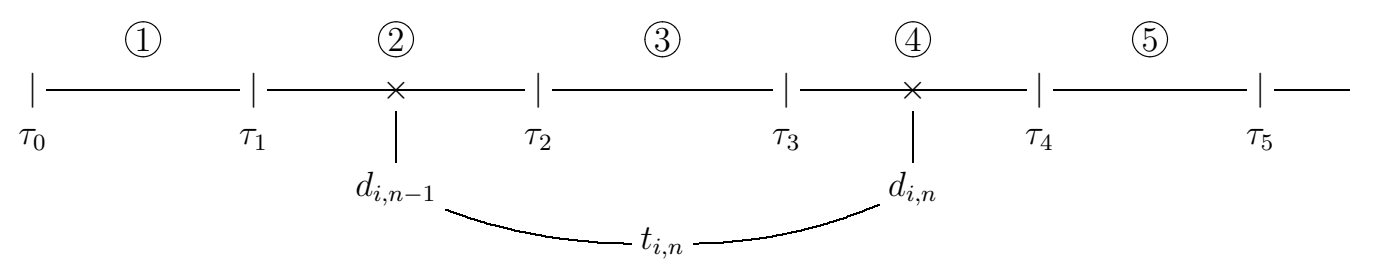

Figure 1: Graphical representation of purchase occasion $d_{i, n}$, interpurchase time $t_{i, n}$ and time indexes of changes in covariates $\tau_{l}$

The hazard function for the $n$-th interpurchase time for household $i$ is denoted by $\lambda_{\text {in }}(t)$, where $t=0$ corresponds with the start of the interpurchase spell. As the basic building block of the model we use a general hazard function $g\left(t ; w_{i n}(t)\right)$, where $w_{i n}(t)$ 
denotes the explanatory variables at duration $t$ associated with the $n$-th interpurchase time. The specification of $g(\cdot)$ depends on the type of hazard model chosen, for example the proportional hazard may look like

$$
g\left(t ; w_{\text {in }}(t)\right)=\exp \left(w_{\text {in }}(t)^{\prime} \gamma\right) \lambda_{0}(t)
$$

where $\lambda_{0}(t)$ is a baseline hazard function, see Gupta (1991) for a similar approach. In this specification the sign of $\gamma$ gives the direction of the effect of an increase in $w_{\text {in }}(t)$ on the hazard. That is, if $\gamma>0$ an increase in $w_{i n}(t)$ results in a decrease of the expected interpurchase time.

The brand preferences of household $i$ at duration $t$ associated with the $n$-th purchase time is denoted by $y_{i n}(t)=y_{i, K_{i n}(t)}$. Note that we impose that the brand preferences are constant during weeks. Although one could consider smaller time intervals to allow for more frequent changes in preference, the (discrete) preference process, by definition, cannot develop in continuous time.

It is obvious how household-specific variables can be included in the hazard specification (1). However, if one wants to include marketing instruments in the model, it is unclear which brand's marketing-mix variables or which combination of brand-specific variables should be included in $w_{i n}(t)$ as brand choice is only revealed at purchase occasions and not in between. Below we present several possibilities to solve this problem. For simplicity of notation we will assume that the model only includes marketing instruments. Other types of explanatory variables can be included in the usual way.

\section{Choice share weighted average of marketing mix}

One may weigh the marketing mix over the $J$ brands using observed market shares as in Gupta (1991). Hence, we have

$$
\lambda_{i n}(t)=g\left(t ; \sum_{j=1}^{J} c_{i j} x_{i n j}(t)\right),
$$

where $c_{i j}$ denotes observed choice share of brand $j$ for household $i$ and $x_{i n j}(t)$ denotes the marketing mix of brand $j$ experienced by household $i$ at time $t$ of the $n$-th purchase occasion. The values of the $x_{i n j}(t)$ variables typically change on a weekly basis.

The household-specific choice shares are usually estimated using the in-sample purchases. Out-of-sample forecasts would have to be based on the in-sample choice shares. This approach is therefore not useful in case one wants to predict purchase timing of 
households for which no purchase history is available. If this type of forecasting is one of the aims of the analysis, one has to rely on one of the other solutions discussed below.

The model parameters can be estimated by Maximum Likelihood. The likelihood function reads

$$
L=\prod_{i=1}^{I} \prod_{n=1}^{N_{i}} L_{i n}
$$

where $L_{i n}$ denotes the likelihood contribution of the $n$-th purchase of household $i$. The likelihood contribution of the $n$-th interpurchase time follows from standard duration theory, see for example Kiefer (1988), and is given by

$$
L_{i n}=\lambda_{i n}\left(t_{i n}\right) S_{i n}\left(t_{i n}\right)=g\left(t_{i n} ; \sum_{j=1}^{J} c_{i j} x_{i n j}\left(t_{i n}\right)\right) \exp \left(-\int_{0}^{t_{i n}} g\left(s ; \sum_{j=1}^{J} c_{i j} x_{i n j}(s)\right) d s\right),
$$

where $S_{i n}(t)=\exp \left(-\int_{0}^{t} \lambda_{i n}(s) d s\right)$ denotes the survivor function.

\section{Inclusive value}

Another frequently used approach is to include the inclusive value from a brand choice model as an explanatory variable in the hazard function as in Chintagunta and Prasad (1998). To describe brand choice we consider a multinomial logit model

$$
\operatorname{Pr}\left[Y_{i n}(t)=j\right]=\frac{\exp \left(\alpha_{j}+x_{i j n}(t)^{\prime} \beta\right)}{\sum_{s=1}^{J} \exp \left(\alpha_{s}+x_{i s n}(t)^{\prime} \beta\right)},
$$

where $Y_{i n}(t)$ denotes the brand choice of household $i$ for the $n$-th purchase occasion, $\alpha_{J}=0$ for identification, and where $\beta$ measures the effect of the marketing mix on brand choice. For simplicity we again assume that there are no other explanatory variables besides the marketing mix. Note that the household only makes a purchase in some of the periods. Therefore, $Y_{i n}(t)$ is only observed at purchase occasions. Although one only observes $Y_{\text {in }}(t)$ at purchase occasions, we assume that the choice probabilities do represent the household's preference for the brands at every point in time.

The inclusive or category value is defined by

$$
I_{i n}(t)=\log \left(\sum_{j=1}^{J} \exp \left(\alpha_{j}+x_{i n j}(t)^{\prime} \beta\right)\right) .
$$

This expression has the interpretation of the expected maximum utility over all brands in the category. The inclusive value is added to the hazard function as explanatory variable. 
Note that the hazard function will be constant over periods of time. The hazard function is in this case given by $\lambda_{i n}(t)=g\left(t ; I_{i n}(t)\right)$.

Again, this model can be estimated by Maximum Likelihood. The likelihood contribution of the $n$-th interpurchase spell of length $t_{i n}$ resulting in a purchase of brand $y_{i n}\left(t_{i n}\right)$ for this specification reads

$$
\begin{aligned}
L_{i n} & =\operatorname{Pr}\left[Y_{i n}\left(t_{i n}\right)=y_{i n}\left(t_{i n}\right)\right] \times \lambda_{i n}\left(t_{i n}\right) S_{i n}\left(t_{i n}\right) \\
& =\operatorname{Pr}\left[Y_{i n}\left(t_{i n}\right)=y_{i n}\left(t_{i n}\right)\right] \times g\left(t_{i n} ; I_{i n}\left(t_{i n}\right)\right) \exp \left(-\int_{0}^{t_{i n}} g\left(s ; I_{i n}(s)\right) d s\right) .
\end{aligned}
$$

The brand choice probability enters the likelihood contribution as the inclusive value is obtained from a brand choice model.

If one decides to model purchase incidence using a binary logit specification, the inclusion of an inclusive value can also be justified as a nested logit model specification, see for example Ben-Akiva and Lerman (1985), Franses and Paap (2001) and Train (2003). In this specification, the inclusive value captures the correlation between the purchase timing and brand choice decision. This approach is followed by for example Ailawadi and Neslin (1998) and Bell et al. (1999).

\section{Preference weighted average of the marketing mix}

An alternative to the choice share approach is to use a weighted average of the marketing mix, where the weighting scheme follows from choice/preference probabilities $\operatorname{Pr}\left[Y_{i n}(t)=\right.$ $j$ ] at time $t$. For this weighting scheme, the hazard specification is given by

$$
\lambda_{i n}(t)=g\left(t ; \sum_{j=1}^{J} \operatorname{Pr}\left[Y_{i, n}(t)=j\right] x_{i n j}(t)\right),
$$

The advantage of this approach over using choice shares as weights, is that this method allows the weights to evolve over time. Changes in preferences, for example due to promotions, are therefore accounted for in this weighting scheme. Additionally, this approach can be used to construct out-of-sample weights for households with unknown purchase history. 


\section{Preference weighted average of hazards}

Instead of taking a weighted average of the marketing mix, one may also consider a weighted average of brand-specific hazard functions

$$
\lambda_{i n}(t)=\sum_{j=1}^{J} \operatorname{Pr}\left[Y_{i n}(t)=j\right] g\left(t ; x_{i n j}(t)\right)
$$

This specification is very similar to the previous one where the weighting occurs inside the hazard function $g(\cdot)$. However due to the nonlinearity of the hazard function it will give different results.

The likelihood function for the weighted hazards and weighted marketing mix have the same form, that is,

$$
L_{i, n}=\operatorname{Pr}\left[Y_{i n}\left(t_{i n}\right)=y_{i n}\left(t_{i n}\right)\right] \times \lambda_{i n}\left(t_{i n}\right) \exp \left(-\int_{0}^{t_{i n}} \lambda_{i n}(s) d s\right)
$$

where either (8) or (9) is used for $\lambda_{i n}(t)$.

\section{Latent preference purchase timing model}

Instead of using brand choice probabilities as convenient weights, it seems more sensible to integrate the duration model and the brand choice model. We assume that the brand choice of a household in a certain week is observable if a household makes a purchase in the product category. During non-purchase weeks, we do not observe brand choice but we assume that households do have a preferred brand. The preferred brand choice is treated as a latent variable and takes the role of the brand choice.

To explain the model, consider the hypothetical situation where we know the preferred brands of household in all weeks, including those where no purchase is made. Assume that the preferred brand in week $k$ is given by $y_{i k}$ and that the hazard function in this week is given by $\lambda\left(t \mid Y_{i k}=y_{i k}\right)$. The brand choice probabilities are given by the logit probabilities $\operatorname{Pr}\left[Y_{i k}=y_{i k}\right]$. The joint density function of a duration from $d_{i, n-1}$ to $d_{i n}$ and preferred brands $y_{i k}$ for weeks $k=K_{i n}(0), \ldots, K_{i n}(t)$ is given by

$$
f\left(t,\left\{y_{i k}\right\}_{k=K_{i n}(0)}^{K_{i n}(t)}\right)=\lambda\left(t \mid Y_{i, K_{i n}(t)}=y_{i, K_{i n}(t)}\right) S\left(t \mid\left\{y_{i k}\right\}_{k=K_{i n}(0)}^{K_{i n}(t)}\right) \prod_{k=K_{i n}(0)}^{K_{i n}(t)} \operatorname{Pr}\left[Y_{i k}=y_{i k}\right] .
$$

In practice, the marketing-mix variables are constant during a week. We assume that the brand preferences given the marketing mix are also constant during a week. Given these 
assumptions, we can expand the survivor function to obtain

$$
\begin{aligned}
& f\left(t,\left\{y_{i k}\right\}_{k=K_{i n}(0)}^{K_{i n}(t)}\right)= \\
& \lambda\left(t \mid Y_{i, K_{i n}(t)}=y_{i, K_{i n}(t)}\right) \operatorname{Pr}\left[Y_{i, K_{i n}(t)}=y_{i, K_{i n}(t)}\right] \exp \left(-\int_{\tau_{K_{i n}(t)-1}-d_{i, n-1}}^{t} \lambda\left(v \mid y_{i, K_{i n}(t)}\right) d v\right) \times \\
& \operatorname{Pr}\left[Y_{i, K_{i n}(0)}=y_{i, K_{i n}(0)}\right] \exp \left(-\int_{0}^{\tau_{K_{i n}(0)}-d_{i, n-1}} \lambda\left(v \mid y_{i, K_{i n}(0)}\right) d v\right) \times \\
& \prod_{k=K_{i n}(0)+1}^{K_{i n}(t)-1} \operatorname{Pr}\left[Y_{i k}=y_{i k}\right] \exp \left(-\int_{\tau_{k-1}-d_{i, n-1}}^{\tau_{k}-d_{i, n-1}} \lambda\left(v \mid y_{i k}\right) d v\right)
\end{aligned}
$$

The first part of (12) refers to the week in which the purchase is made, the middle part concerns the period of the start of the duration to the first change in the marketing mix. The third part of (12) deals with all other periods of constant preferences and marketing mix.

So far we have assumed that we know the preferred brands, even at weeks where there is no purchase at all. Of course, we do not observe brand preferences at weeks without purchases. Hence, we have to sum over all possible realizations of the latent brand preferences in these weeks to obtain the joint density of the interpurchase time and the brand choice at the purchase occasion. Hence, we sum (12) over all possible values of $y_{i k}$ in weeks $k=K_{i n}(0), \ldots, K_{i n}(t)-1$, that is,

$$
\begin{aligned}
& f\left(t, y_{i, K_{i n}(t)}\right)=\sum_{y_{i, K_{i n}(0)}=1}^{J} \ldots \sum_{y_{i, K_{i n}(t)-1}=1}^{J} f\left(t,\left\{y_{i k}\right\}_{k=K_{i n}(0)}^{K_{i n}(t)}\right) \\
& =\lambda\left(t \mid Y_{i, K_{i n}(t)}=y_{i, K_{i n}(t)}\right) \operatorname{Pr}\left[Y_{i, K_{i n}(t)}=y_{i, K_{i n}(t)}\right] \exp \left(-\int_{\tau_{K_{i n}(t)-1}-d_{i, n-1}}^{t} \lambda\left(v \mid y_{i, K_{i n}(t)}\right) d v\right) \times \\
& \quad \sum_{y_{i, K_{i n}(0)=1}^{J}}^{J} \operatorname{Pr}\left[Y_{i, K_{i n}(0)}=y_{i, K_{i n}(0)}\right] \exp \left(-\int_{0}^{\tau_{K_{i n}(0)}-d_{i, n-1}} \lambda\left(v \mid y_{i, K_{i n}(0)}\right) d v\right) \times \\
& \prod_{k=K_{i n}(0)+1}^{K_{i n}(t)-1}\left(\sum_{j=1}^{J} \operatorname{Pr}\left[Y_{i k}=j\right] \exp \left(-\int_{\tau_{k-1}-d_{i, n-1}}^{\tau_{k}-d_{i, n-1}} \lambda\left(v \mid y_{i k}\right) d v\right)\right)
\end{aligned}
$$

The likelihood contribution $L_{i n}$ of the $n$-th interpurchase time of household $i$ resulting in a purchase of brand $y_{i, K_{i n}\left(t_{i n}\right)}$ now equals $f\left(t_{i n}, y_{i, K_{i n}\left(t_{i n}\right)}\right)$. 


\section{Empirical comparison}

In this section we compare the various model specifications for the interpurchase timing model using household panel scanner data. For three different categories of fast-moving consumer goods we will estimate the five different specifications discussed in Section 2. The performance of the different specifications is measured using in-sample and out-ofsample criteria.

The data we use is part of the so-called ERIM database, which is collected by A.C. Nielsen. The data span the years 1986 to 1988, and the particular subset we use concerns purchases of catsup, laundry detergent and yogurt by households in Sioux Falls (South Dakota, USA). We split the data sets in two parts such that the number of households is roughly the same in both samples. The first part is used to estimate the parameters of the various models, while the second part is used for out-of-sample evaluation. Table 1 provides an overview of the number of brands, households and number of purchases in the samples. The catsup category contains the brands Del Monte, Heinz and Hunts, the yogurt category contains Dannon, Nordica, W-B-B, Yoplait and a rest brand, while for the detergent category we have Cheer, Oxydol, Surf, Tide, Wisk and a rest brand.

- Insert Table 1 about here-

We use a standard multinomial logit model to describe brand choice and to describe interpurchase timing we use a proportional hazard model with a log-logistic baseline hazard, to be more precise the baseline hazard reads

$$
\lambda_{0}(t)=\frac{\alpha \gamma t^{\alpha-1}}{1+\gamma t^{\alpha}}
$$

where $\alpha>0$ and $\gamma>0$. This specification allows the baseline hazard to be monotonically decreasing or inverted U-shaped. Chintagunta and Haldar (1998) show that for modeling purchase timing this baseline hazard outperforms commonly used alternatives as the Weibull or Erlang-2 specification. The multinomial logit model we use to model brand choice contains brand-specific intercepts, the marketing mix of all brands in the market (price, display and feature) and a lagged brand choice dummy capturing statedependence. As explanatory variables in the hazard model we use household size and household income as these variables are known to influence interpurchase timing. To control for inventory effects, such as stockpiling, we use the volume previously bought in the category as an additional variable, see also Chintagunta and Prasad (1998) for a 
similar approach. These variables are household specific and therefore are not subject to the difficulties presented in this paper for brand-specific variables. Finally, we use the available marketing instruments, that is, price, display and feature. The five different ways to include the marketing mix in the hazard specification discussed in Section 2 lead to five alternative models.

In this paper we are not so much interested in specific values of estimated parameters, the focus lies on the comparison of the various model specifications. To this end the analysis is split up in two parts. First, we analyze the differences in descriptive power, where we do not allow for unobserved heterogeneity in the brand choice model. In this case the model specification using individual choice shares to obtain a category average of the marketing efforts of the different brands in a category (2) clearly has an advantage over the other specifications. It allows for an easy representation of between-household heterogeneity in brand preferences. Differences in brand preferences will have a large influence on the relative importance of the marketing mix of individual brands on the purchase incidence decision. We expect this specification to be superior in in-sample fit. For out-of-sample prediction, individual choice shares may not be available if we consider households outside the estimation sample. One may use the in-sample average choice share across households as a predictor for the out-of-sample individual choice shares. In this case the forecasting performance of the individual choice share specification will probably be lower.

Concerning in-sample performance we expect that explicit modeling of (unobserved) heterogeneity in brand preferences will lead to the same or even better fit of the alternative models compared to the specification based on choice shares. This assertion is analyzed in the second part of this section.

\section{No unobserved heterogeneity}

First of all we compare the performance of the different specifications without controlling for unobserved heterogeneity. Table 2 shows some performance statistics of six models for the three categories under investigation. As in-sample measures we consider the maximum $\log$ likelihood value, the AIC and the BIC. The value of the log likelihood function for the out-of-sample observations evaluated at the estimate based on the in-sample observations is used to evaluate the forecasting performance of the various model specifications. For the choice share specification we consider two values of the out-of-sample log likelihood. The first is based on household-specific choice shares estimated using out-of-sample ob- 
servations, while for the second the choice shares are set to the in-sample average choice shares. This measure represents the case in which choice share information is not available when forecasting interpurchase times.

- Insert Table 2 about here -

Table 2 displays an overview of the results. Several conclusions can be drawn from this table. First, if we consider in-sample measures, the specification that uses the householdspecific choice shares as weights performs best for all criteria. Note that we did not count the choice shares as parameters in computing the information criteria, although strictly speaking these estimated shares are to be seen as parameters. If we would count the weights as parameters, the choice-share specification would be the lowest in rank on the $\mathrm{AIC}$ and BIC measures. Secondly, the inclusive value specification turns out to perform worst on all measures. Finally, if we ignore the choice share specification, the latent preference model performs best for all performance measures.

If we consider out-of-sample measures we notice the same pattern. The only difference is that for the catsup category both the weighted hazard and the weighted marketing-mix specifications outperform the latent preference model. Furthermore, if we compute the out-of-sample log likelihood value using an average of in-sample household-specific choice shares, the forecasting performance of the "choice share model" is almost always worse than of the other specifications, with the exception of the inclusive value specification for the catsup category.

\section{Unobserved heterogeneity}

We have seen that the model based on household-specific choice shares performs best on in-sample and out-of-sample measures. As already discussed before, we expect this superiority to vanish if we explicitly model heterogeneity in brand preference among households. To validate this claim, we estimate the models while allowing for unobserved heterogeneity in brand preferences and the average purchase rate. That is, we allow the brand intercepts and the intercept of the hazard function to differ across households through the use of latent segments, see Wedel and Kamakura (1999).

To reduce the probability of ending up in a local maximum of the likelihood, we estimate the heterogeneous models with ten different starting values. The results below are based on the best of these ten starting values. 
To summarize the results we only compare the performance of the individual choice share model with the latent preference model in detail. Note that the latent preference model turned out to be second best in the homogeneous case. Table 3 provides an overview of the results for the three product categories. If we correct for unobserved heterogeneity the relative performance of the latent preference model versus the model based on choice shares indeed improves. As we only want to illustrate that the latent preference model with unobserved heterogeneity can outperform the choice share model, we stop adding segments when this goal is reached in-sample as well as out-of-sample. We see that with 6 segments the latent preference model outperforms the choice share model both in-sample as out-of-sample for all three categories. Unreported results show that similar results are found for all other suggested specifications, except for the inclusive value model for catsup. For this category the inclusive value model has the worst in-sample performance for all segments. For one to five segments the out-of-sample performance is also worst of all, when six segments are considered the inclusive value model performs better than the choice share specification.

- Insert Table 3 about here -

To analyze the relative performance of all specifications in case of unobserved heterogeneity, we consider the detergent category in more detail. As can be seen from Table 3, for this category it holds that up to two segments the specification based on choice shares outperforms the latent preference model on the basis of in-sample log likelihood value. In case three or more segments are used the advantage of the household-specific choice shares is compensated by the heterogeneity captured by the part of the model that captures brand choice. In Table 4 we present the in-sample and out-of-sample log likelihood value for the detergent category for all non-choice share models. We conclude that the latent preference model performs relatively best for most number of segments. Only when four segments are used it is beaten by the specification based on a weighted marketing mix. This last specification however performs worst for three segments. The out-of-sample performance measures show a similar pattern.

- Insert Table 4 about here -

To check whether these results also hold for the other two categories, we provide in Table 5 an overview of the performance of the non-choice share based models. To prevent that our results are influenced by the number of segments imposed, we report in the final 
column of the table the average rank of the model across the three product categories for the different segment sizes. If we consider the in-sample measures, the latent preference model specification performs best for all segment sizes. For out-of-sample measures the latent preference model is best or second best. The final column of the table shows the overall rank. We see that the overall rank of the latent preference model is best and that the inclusive value specification has the largest average rank value. This result holds for in-sample as well as out-of-sample performance.

- Insert Table 5 about here -

\section{Conclusions}

In this paper we have considered the practical question of what to do with brand-specific marketing efforts when modeling interpurchase timing. As purchase timing is measured on the category level, one has to somehow aggregate the brand level information. In the literature there are two popular techniques. Category marketing efforts are often formed by calculating a weighted average of the marketing mix of individual brands. As weights household-specific choice shares are often used. Another approach is to summarize all marketing-mix variables of all brands into the so-called inclusive value.

We have proposed three alternative specifications. For the first alternative we create category level marketing instruments using household-specific weights that are obtained from a brand choice model. The second alternative uses the same weights to aggregate over brand-specific incidence probabilities (or hazards). Finally we suggested a specification that integrates a brand choice model with the purchase timing.

In an empirical comparison of the resulting five specifications for three categories of fast-moving consumer goods, we find that when unobserved heterogeneity is not accounted for the specification using choice shares performs best. However, this specification is less useful for out-of-sample forecasting as in this case household's choice shares are in general unknown. For out-of-sample forecasting the latent preference model tends to perform best. If unobserved heterogeneity is accounted for the latent preference model also performs best in sample.

We conclude with a practical summary of the results. If one is only interested in describing purchase timing and not in brand choice, one obtains the best performance by weighting the marketing mix in the interpurchase time model using individual choice 
shares. However, if one wants to use the model for out-of-sample forecasting and out-ofsample individual choice shares are unknown, one has to use one of the other models. In that case, information from a brand choice model can be used to weight brand-specific marketing efforts for the interpurchase model. These models outperform the choice share based model if one explicitly models the unobserved heterogeneity in the brand choices. The overall performance of the latent preference model where one integrates brand choice and interpurchase timing is best, although the differences with the weighted marketing mix and weighted hazard specification are sometimes not substantial. However, the latent preference model outperforms the inclusive value specification. 
Table 1: Data characteristics of three categories of fast-moving consumer goods

Category No. brands No. households No. purchases

In-sample Out-of-sample In-sample Out-of-sample

$\begin{array}{llllll}\text { Catsup } & 3 & 363 & 356 & 3742 & 3610 \\ \text { Detergent } & 6 & 303 & 295 & 2318 & 2080 \\ \text { Yogurt } & 5 & 210 & 209 & 4337 & 3605\end{array}$


Table 2: Performance measures of different interpurchase models without correcting for unobserved heterogeneity ${ }^{1}$

\begin{tabular}{|c|c|c|c|c|c|c|}
\hline \multicolumn{2}{|c|}{ choice model } & $\begin{array}{l}\text { choice } \\
\text { shares }^{2}\end{array}$ & $\begin{array}{l}\text { Dura } \\
\text { inclusive } \\
\text { value }\end{array}$ & $\begin{array}{l}\text { tion/Choice } \\
\text { weighted } \\
\text { hazard }\end{array}$ & $\begin{array}{l}\text { models } \\
\text { weighted } \\
\text { mark. mix }\end{array}$ & $\begin{array}{c}\text { latent } \\
\text { preferences }\end{array}$ \\
\hline \multicolumn{7}{|c|}{ Catsup category } \\
\hline $\log L$ & -1909.36 & $\underline{-13872.4}$ & -13892.5 & -13883.6 & -13881.9 & -13878.2 \\
\hline AIC & 3830.72 & $\underline{27775.9}$ & 27810.9 & 27799.2 & 27795.8 & 27788.3 \\
\hline $\mathrm{BIC}$ & 3854.08 & $\underline{27838.3}$ & 27861.6 & 27861.5 & 27858.1 & 27850.6 \\
\hline $\begin{array}{l}\text { out-of-sample } \log L \\
\text { with in-sample shar }\end{array}$ & $\begin{array}{l}-1541.13 \\
\text { ares }\end{array}$ & $\frac{-13072.1}{-13094.1}$ & -13110.4 & $\underline{-13092.3}$ & -13093.1 & -13095.0 \\
\hline
\end{tabular}

\begin{tabular}{lr|rrrrr}
\multicolumn{7}{c}{ Detergent category } \\
$\log L$ & -2335.37 & $\underline{-8996.27}$ & -9012.75 & -9004.35 & -9004.11 & -8998.17 \\
AIC & 4688.73 & $\underline{18030.5}$ & 18057.5 & 18046.7 & 18046.2 & 18034.3 \\
out-of-sample $\log L$ & -2254.69 & $\underline{-8294.79}$ & -8317.58 & -8312.68 & -8312.20 & $\underline{-8311.87}$ \\
\multicolumn{2}{l}{ with in-sample shares } & -8318.39 & & & &
\end{tabular}

\begin{tabular}{lr|rrrrr}
\multicolumn{7}{c}{ Yogurt category } \\
$\log L$ & -3869.15 & $\underline{-13277.2}$ & -13414.7 & -13399.0 & -13402.0 & -13387.3 \\
AIC & 7754.30 & $\underline{26590.4}$ & 26859.5 & 26834.0 & 26840.0 & 26810.6 \\
BIC & 7784.01 & $\underline{26657.2}$ & 26915.2 & 26900.8 & 26906.8 & 26877.5 \\
out-of-sample $\log L$ & -3707.60 & $\underline{-12372.7}$ & -12408.4 & -12398.6 & -12397.1 & $\underline{-12387.7}$ \\
\multicolumn{2}{l}{ with in-sample shares } & -12425.1 & & & &
\end{tabular}

${ }^{1}$ Underlined entries indicate the best performing model, per performance measure. For the out-ofsample likelihood the best performing model based on in-sample shares is also underlined.

2 The interpurchase timing model using choice shares can be estimated independently from the brand choice model. To allow for easy comparison, the performance statistics however show the results of the combination of the duration model and the brand choice model. 
Table 3: Likelihood differences Latent preference model - Choice shares model (average in-sample shares used for out-of-sample choice shares)

\begin{tabular}{llcccc}
\multicolumn{5}{c}{ Number of segments } \\
1 & 2 & 3 & 4 & 5 & 6
\end{tabular}

In-sample log-likelihood difference

$\begin{array}{lrrrrrr}\text { Catsup } & -5.84 & -12.10 & -13.50 & -9.90 & -13.50 & 1.30 \\ \text { Yogurt } & -110.15 & 6.80 & -5.00 & -6.90 & 48.92 & 35.20 \\ \text { Detergent } & -1.90 & -3.27 & 13.08 & 28.34 & 35.90 & 13.33\end{array}$

Out-of-sample log-likelihood difference

$\begin{array}{lrrrrrr}\text { Catsup } & -0.87 & -3.70 & 3.40 & 6.20 & 6.70 & 53.40 \\ \text { Yogurt } & 37.40 & -51.80 & 119.10 & 99.30 & 119.00 & 138.49 \\ \text { Detergent } & 6.52 & 12.33 & 47.14 & 2.83 & 44.65 & 9.35\end{array}$


Table 4: Performance measures for interpurchase time models with unobserved heterogeneity for the detergent category (largest likelihood value per number of segments in boldface)

Number of segments

$\begin{array}{llllll}1 & 2 & 3 & 4 & 5 & 6\end{array}$

In-sample $\log L$

Choice model $-2335.37 \quad-2213.76 \quad-2133.46 \quad-2085.64 \quad-2046.62 \quad-2016.14$

Inclusive value $\begin{array}{llllll}-9012.75 & -8680.78 & -8582.07 & -8522.32 & -8452.02 & -8423.58\end{array}$

Weighting hazard $\begin{array}{llllll}-9004.35 & -8677.84 & -8583.75 & -8519.96 & -8459.35 & -8418.73\end{array}$

Weighting mark. mix

$\begin{array}{llllll}-9004.11 & -8677.62 & -8596.64 & -8513.10 & -8449.39 & -8421.50\end{array}$

Latent preferences

-8998.17 -8673.07 -8578.36 -8516.65 -8443.07 -8410.93

Out-of-sample $\log L$

choice model

Inclusive value

Weighting hazard

Weighting mark. mix

Latent preferences $\begin{array}{lllllll}-2254.69 & -2195.01 & -2132.77 & -2115.76 & -2077.77 & -2071.70\end{array}$

$\begin{array}{llllll}-8317.58 & -8164.67 & -8120.61 & -8113.00 & -8084.83 & -8058.41\end{array}$

$\begin{array}{llllll}-8312.68 & -8154.85 & -8124.28 & -8097.82 & -8073.05 & -8063.59\end{array}$

$\begin{array}{llllll}-8312.20 & -8154.02 & -8149.01 & \mathbf{- 8 0 8 6 . 9 0} & -8042.93 & \mathbf{- 8 0 4 2 . 3 6}\end{array}$

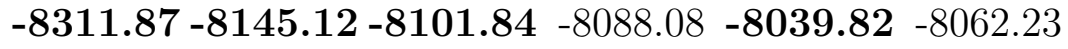


Table 5: Average ranks of model performance over three categories (excluding choice shares specification)

$\begin{array}{lllllll}1 & 2 & 3 & 4 & 5 & 6 & \text { overall }\end{array}$

\section{In-sample average rank}

$\begin{array}{lccccccc}\text { Inclusive value } & 4.00 & 3.33 & 2.33 & 3.00 & 2.67 & 4.00 & 3.22 \\ \text { Weighted hazard } & 2.67 & 3.33 & 3.33 & 3.33 & 3.33 & 2.67 & 3.11 \\ \text { Weighted mark. mix } & 2.33 & 2.33 & 3.00 & 2.00 & 2.67 & 2.33 & 2.44 \\ \text { Latent preferences } & 1.00 & 1.00 & 1.33 & 1.67 & 1.33 & 1.00 & 1.22\end{array}$

\section{Out-of-sample average rank}

$\begin{array}{llllllll}\text { Inclusive value } & 4.00 & 3.67 & 3.33 & 4.00 & 4.00 & 3.33 & 3.72\end{array}$

$\begin{array}{llllllll}\text { Weighted hazard } & 2.33 & 2.33 & 2.67 & 2.67 & 2.33 & 2.67 & 2.50\end{array}$

$\begin{array}{llllllll}\text { Weighted mark. mix } & 2.00 & 2.00 & 3.00 & 1.33 & 2.33 & 1.67 & 2.06\end{array}$

$\begin{array}{llllllll}\text { Latent preferences } & 1.67 & 2.00 & 1.00 & 2.00 & 1.00 & 2.33 & 1.67\end{array}$ 


\section{References}

Ailawadi, K. L. and S. A. Neslin (1998), The Effect of Promotion on Consumption: Buying More and Consuming it Faster, Journal of Marketing Research, 35, 390-398.

Bell, D. R., J. Chiang, and V. Padmanabhan (1999), The Decomposition of Promotional Response: An Empirical Generalization, Marketing Science, 18, 504-526.

Ben-Akiva, M. and S. R. Lerman (1985), Discrete Choice Analysis: Theory and Application to Travel Demand, vol. 9 of MIT Press Series in Transportation Studies, MIT Press, Cambridge (MA).

Bucklin, R. E. and S. Gupta (1992), Brand Choice, Purchase Incidence, and Segmentation: An Integrated Approach, Journal of Marketing Research, 29, 201-215.

Chintagunta, P. K. and S. Haldar (1998), Investigating Purchase Timing Behavior in Two Related Product Categories, Journal of Marketing Research, 35, 43-53.

Chintagunta, P. K. and A. R. Prasad (1998), An Empirical Investigation of the "Dynamic McFadden" Model of Purchase Timing and Brand Choice: Implications for Market Structure, Journal of Business \& Economic Statistics, 16, 2-12.

Doornik, J. A. (1999), Object-Oriented Matrix Programming Using Ox, 3rd edn., London: Timberlake Consultants Press and Oxford: www.nuff.ox.ac.uk/Users/Doornik.

Franses, P. H. and R. Paap (2001), Quantitative Models in Marketing Research, Cambridge University Press, Cambridge.

Gupta, S. (1988), Impact of Sales Promotions on When, What, and How Much to Buy, Journal of Marketing Research, 25, 342-355.

Gupta, S. (1991), Stochastic Models of Interpurchase Time With Time-Dependent Covariates, Journal of Marketing Research, 28, 1-15.

Helsen, K. and D. C. Schmittlein (1993), Analyzing Duration Times in Marketing: Evidence for the Effectiveness of Hazard Rate Models, Marketing Science, 11, 395-414.

Jain, D. C. and N. J. Vilcassim (1991), Investigating Household Purchase Timing Decisions: A Conditional Hazard Function Approach, Marketing Science, 10, 1-23. 
Kiefer, N. M. (1988), Economic Duration Data and Hazard Functions, Journal of Economic Literature, 26, 646-679.

Seetharaman, P. B. and P. K. Chintagunta (2003), The Proportional Hazard Model for Purchase Timing: A Comparison of Alternative Specifications, Journal of Business 8 Economic Statistics, forthcoming.

Train, K. E. (2003), Discrete Choice Models with Simulation, Cambridge University Press, Cambridge.

Vilcassim, N. J. and D. C. Jain (1991), Modeling Purchase-Timing and Brand-Switching Behavior Incorporating Explanatory Variables and Unobserved Heterogeneity, Journal of Marketing Research, 28, 29-41.

Wedel, M. and W. A. Kamakura (1999), Market Segmentation: Conceptual and Methodological Foundations, Kluwer Academic Publishers, Dordrecht. 